\title{
Core modes as a seismic probe of mixing beyond the convective core
}

\author{
B. L. Popielski \\ Warsaw University Observatory, Al. Ujazdowskie 4, 00-478, Warsaw, Poland
}

The debate on the extent of mixing beyond the convective core is not yet settled. For intermediate mass stars, $1.2-2 \mathrm{M}_{\odot}$, where the convective core tends to grow, three mixing recipes must be considered. These are: the layered model (LY; Spruit 1992), which assumes no mixing, the semi-convective model (SC; Schwarzschild \& Härm 1958), where moderate mixing occurs, and the overshooting model (OV), where the extent is the largest and may be suitably chosen. There is a subset of stochastically excited modes which is particularly useful for sounding the partially mixed layers above the core. I will refer to this subset as core modes because these modes are partially trapped in the g-cavity encompassing the inhomogeneous layers. The consecutive core modes $(k=1,2$, etc.) are nearly equidistant in period. The $\ell=1$ core modes are expected to have larger surface amplitudes compared to the modes of higher degree, not only near the avoided crossing frequency and hence should be easily detected.

I tested whether the mixing recipes may be distinguished with the use of core modes. For testing I chose $\eta$ Boo, for which the possible presence of core modes was suggested (Di Mauro et al. 2003). I looked for such modes in the rich oscillation spectrum from MOST (Guenther et al. 2005). Possible dipole modes were identified by their large departure from the $\ell=1$ p-mode ridge in the echelle diagram. I identified 7 possible dipole core modes. Only model calculations could be used to state which of these modes are dipole core modes.

I calculated evolutionary models of $\eta$ Boo for three mixing recipes. The $0.1 H_{p}$ overshooting distance was adopted. The models were constrained by the following non-seismic observables: effective temperature, $T_{\text {eff }}$, luminosity, $L$, surface metallicity, $(Z / X)_{s}$ and two seismic parameters derived from the $\ell=0$ ridge in the echelle diagram: the mean large separation, $\bar{D}$, and the width of the ridge, $\Delta \nu_{D}$. In these models there are core modes up to $k=3$ (see Table 1). However, our identification procedure could have eliminated the core modes, whose frequencies were too close to the p-mode ridge, from the observed spectrum. Thus, we should expect at most 3 dipole core modes. The remaining peaks could be attributed to modes of higher $\ell$ or just artifacts.

Identification of possible core modes was done using the $\Pi-\Delta \Pi$ diagram, where $\Pi$ stands for period and $\Delta \Pi$ stands for the period difference between two consecutive modes. I identified 7 pairs of peaks as $k=1$ or $k=2$. If we assume that only two peaks correspond to core modes, we cannot exclude any of the mixing recipes considered because we don't know which 5 peaks have to be rejected. On the other hand, if we assume that three peaks, found to be nearly equidistant in period, correspond to core modes, then the layered model (LY) is favoured. Of course, we don't know which case we deal with. Clearly, the available data do not allow us to distinguish the mixing recipes. In part this is due to ambiguities in the mode identification, which may vanish for a much cleaner spectrum. In part it is due to rather

\begin{tabular}{|c|cc|ccc|}
\hline & $\alpha$ & $t[\mathrm{Gyr}]$ & \multicolumn{3}{|c|}{$\Pi_{1}, \Pi_{2}, \Pi_{3}[\mathrm{~min}]$} \\
\hline $\mathrm{LY}$ & 2.10 & 2.384 & 15.44 & 21.58 & 28.33 \\
$\mathrm{SC}$ & 1.95 & 2.402 & 18.42 & 25.80 & 33.22 \\
$\mathrm{OV}_{0}$ & 1.95 & 2.244 & 23.16 & 32.22 & 39.49 \\
\hline
\end{tabular}

Table 1: Parameters of central models reproducing the observables of $\eta$ Boo for the three mixing recipes. In columns we find the values of the mixing-length parameter, age and periods for the first three dipole core modes. The models have identical mass of $1.71 \mathrm{M}_{\odot}$ and common initial chemical composition, $X_{0}=0.73$ and $Z_{0}=0.034$. 
poor constraints for models, which result in large uncertainties of $\Pi$ and $\Delta \Pi$. This may be improved by reducing uncertainties of temperature, metallicity and oscillation frequencies.

Acknowledgments. I thank W. A. Dziembowski for many valuable discussions. I also thank the European Helio- and Asteroseismology Network HELAS for financial support.

\section{References}

Di Mauro M. P., Christensen-Dalsgaard J., Kjeldsen H., Bedding T. R., Paternò L., 2003, A\&A, 404, 341 Guenther D. B., Kallinger T., Reegen P., et al., 2005, ApJ, 635, 547

Schwarzschild M., Härm R., 1958, ApJ, 128, 348

Spruit H. C., 1992, A\&A, 253, 131

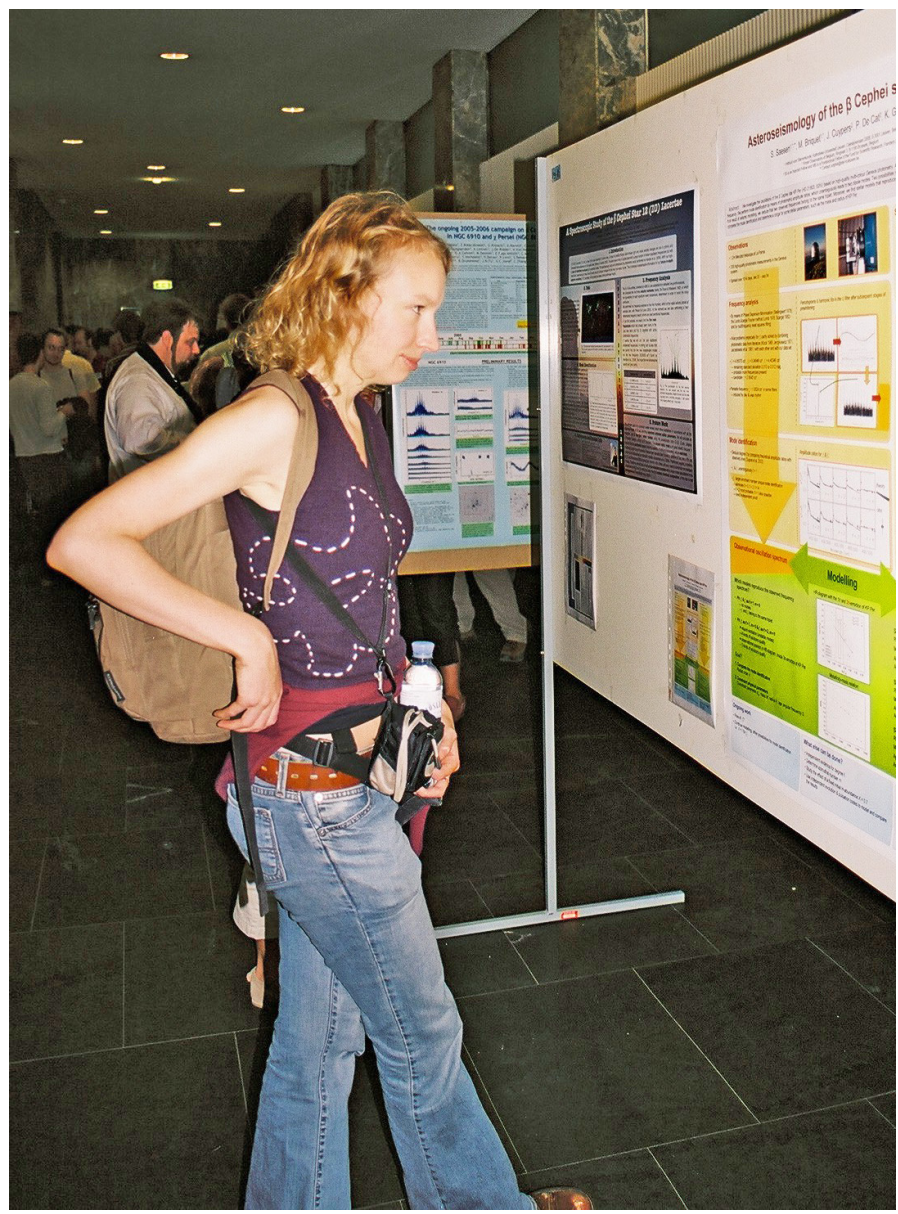

Katrien Kolenberg on close inspection of a poster. 